\title{
Association Between TRPC6 Channel and NLRP3 Inflammasome Activities in Kidney Tissue of Children With Henoch-Schönlein Purpura Nephritis
}

\section{Xianhe Wang}

The First Affiliated Hospital of Anhui Medical University

Man Wu

The First Affiliated Hospital of Anhui Medical University

Yan Liu

The First Affiliated Hospital of Anhui Medical University

Hui Gao

The First Affiliated Hospital of Anhui Medical University

Fang Deng ( $\boldsymbol{V}$ dengfang@ahmu.edu.cn )

The First Affiliated Hospital of Anhui Medical University

\section{Research article}

Keywords: Henoch-Schönlein purpura nephritis, TRPC6, NLRP3 inflammasome, immunohistochemistry, Child

Posted Date: November 12th, 2020

DOl: https://doi.org/10.21203/rs.3.rs-103493/v1

License: (1) This work is licensed under a Creative Commons Attribution 4.0 International License. Read Full License 


\section{Abstract}

\section{Background}

During the development of Henoch-Schönlein purpura nephritis (HSPN), the activation and overexpression of transient receptor potential cation channel protein 6 (TRPC6) and the activation of Nod-like receptor protein 3 (NLRP3) inflammasome play a very important role. However, whether the expression of TRPC6 in children with HSPN is related to the activation of NLRP3 inflammasome has not been reported.

Methods

We obtained kidney biopsy specimens of 33 children with HSPN and 6 controls with renal trauma. Immunohistochemistry was used to detect the expression of TRPC6, NLRP3, ASC, caspase-1, IL-1ß, IL-18. Student's t-test was used to analyze the difference between the HSPN and control group. Pearson correlation test was used to estimate the correlation between TRPC6 and NLRP3 inflammasome among HSPN children. A bootstrap approach was performed for mediation effects of NLRP3 inflammasome on the association between TRPC6 and HSPN.

Results

The expressions of TRPC6, NLRP3, ASC, Caspase-1, IL-1 $\beta$, IL-18 in kidney tissues of children with HSPN were significantly higher than those in the control group (all $P$ values $<0.05$ ). Significant correlations of TRPC6 with NLRP3, ASC, and IL-18 were observed. NLRP3 and ASC were also related to the levels of IL-1 $\beta$ and IL-18. Significant mediation effects $(0.08,95 \% \mathrm{Cl}=0.04-0.15)$ of ASC on the association between TRPC6 and HSPN was revealed, with explaining $58.25 \%$ of the total effects.

Conclusion

TRPC6 may cause the release of IL-1 $\beta$ and IL-18 by activating the NLRP3 inflammasome, especially the expression of ASC, thereby damaging the kidneys of children with HSP.

\section{Background}

Henoch-Schönlein purpura nephritis (HSPN) is a common clinical pediatric disease, mainly with gross or microscopic hematuria, repeated skin purpura, edema, and increased blood pressure as clinical manifestations ${ }^{1}$. HSPN is the most common and serious complication of Henoch-Schönlein purpura (HSP), and the main factor affecting the long-term prognosis of HSP children. It has been reported that 6 to 24 per 100000 children younger than 17 years will develop HSP $^{2}$. However, up to half of HSP children will develop HSPN, $90 \%$ of which will occur within 6 months of onset ${ }^{3,4}$. In China, HSPN has been the second-most common secondary glomerular disease 5 . If not treated in time, it can lead to chronic renal insufficiency in children, which will have a serious impact on the health and quality of children, even on 
their whole life span. Therefore, it is important to clarify the pathogenesis and the clinical manifestations and to ascertain the most appropriate treatment of HSPN.

NOD-like receptor protein 3 (NLRP3) inflammasome is one of the most famous inflammasomes in mammals, and it plays a very important role in innate immunity. The activation of NLRP3 inflammasome is closely related to a variety of inflammation-related diseases, such as type 2 diabetes, atherosclerosis, Alzheimer's disease, and other diseases ${ }^{6}$. Studies have shown that in high glucose or diabetes, the activation of the NLRP3 inflammasome of kidney tissue is the key upstream mechanism of podocyte inflammatory injury ${ }^{7,8}$. Research ${ }^{9}$ pointed out that ion efflux $\left(\mathrm{K}^{+}, \mathrm{Cl}^{-}\right)$, reactive oxygen species (ROS), or mitochondrial DNA (mtDNA) oxidation and NLRP3 post-translational modification (ubiquitination, phosphorylation) can regulate the activation of NLRP3 inflammasome. In addition to bacterial, viral, and fungal infections, more and more endogenous danger signals, such as mitochondrial $\operatorname{ROS}^{10}$ and potassium efflux ${ }^{11}$, maybe activators of NLRP3 inflammasome. The key role of NLRP3 inflammasome in kidney inflammation has been confirmed in a variety of kidney disease models, including I/R injury ${ }^{12,13}$, folic acid nephrotoxicity ${ }^{14}$, caused by rhabdomyolysis $\mathrm{AKI}^{15}$, and contrast-induced $A K \mathrm{KI}^{16}$. However, few studies have explored its role in podocyte damage in immune-related kidney diseases. Transient receptor potential (TRP) channels are a large class of plasma membrane proteins, including TRPC, TRPM, etc. Typical TRPC6 is a non-selective $\mathrm{Ca}^{2+}$ permeable cation channel expressed in many different cell types. Renal tissue TRPC6 channel protein is mainly expressed in the foot process slit membrane ${ }^{17}$, but also in mesangial cells ${ }^{18}$. The TRPC6 channel is associated with the progression of several forms of kidney disease, such as primary and secondary FSGS (familial forms of focal segmental glomerulosclerosis), glomerulosclerosis during autoimmune glomerulonephritis, and type 1 diabetes ${ }^{19}$. Previous research by our research group found that RAS activation in the kidney may be involved in the occurrence and development of kidney damage in children with $\mathrm{HSP}^{20}$. Experiments with nephropathy model rats also indicate that the increased expression of AGT in the kidney is related to the increased production of

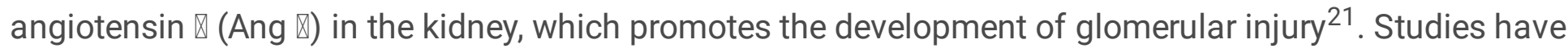
shown that Ang $\triangle$ can quickly activate the TRPC 6 channel of podocytes and mediate calcium influx, and at the same time cause a significant upregulation of TRPC6 activity and expression ${ }^{22,23}$. Experimental evidence showed that calcium channel proteins (e.g., TRPM1, TRPM2) can mediate NLRP3 activation and cause cell damage in other tissues ${ }^{24,25}$. Extracellular $\mathrm{Ca}^{2+}$ influx or $\mathrm{Ca}^{2+}$ release from the intracellular calcium pool to the cytoplasm and cause mitochondrial dysfunction is a common pathway for the activation of NLPRP3 inflammasome by multiple activators ${ }^{26}$. Therefore, we believe that TRPC6 and NLRP3 inflammasome play an important role in the occurrence and development of HSPN.

This study was to investigate the changes in the expression of TRPC6 and NLRP3 inflammasome in kidney tissues of children with HSPN and their relationship.

\section{Materials And Methods}

\subsection{Participants and specimens}


From January 2018 to December 2019, 33 children with HSPN were executed a renal biopsy, when they hospitalized in the Department of Nephrology of the First Affiliated Hospital of Anhui Medical University. 19 males and 14 females were included with an average age of 10 years. All cases met the diagnostic criteria of the Evidence-Based Guidelines for the Diagnosis and Treatment of Henoch-Schönlein purpura Nephritis (2016) by the Nephrology Group of the Pediatric Branch of the Chinese Medical Association ${ }^{27}$. The control group specimens were derived from normal tissues from kidney trauma or tumor surgery. The renal biopsy specimens and those control group specimens were further analyzed in the laboratory. The study was approved by the Medical Research Ethics Committee of the First Affiliated Hospital of Anhui Medical University, informed consent was obtained from all guardians of individual participants included in the study.

\subsection{TRPC6 and NLRP3 inflammasome related indicators detection}

Immunohistochemistry (IHC), the wax block was cut into 4um thick tissue sections, dewaxed the tissue sections, and rehydrated, and then repaired antigen, incubated with $3 \%$ hydrogen peroxide for 15 minutes, blocked with $10 \%$ goat serum for 30 minutes at room temperature. After incubation with the primary antibody (anti-IL-1 $\beta$, anti-IL-18, anti-NLRP3, and anti-Caspase-1 antibodies, Wan Biotechnology Company, 1:200; anti-ASC antibody, Wan Biotechnology Company, 1:150; anti-TRPC6 antibody, Wuhan Sanying Biological Company, 1:200) overnight at $4^{\circ} \mathrm{C}$, washed with phosphate buffered saline (PBS) 3 times, incubated with secondary antibody at room temperature for 50 minutes, and washed with PBS for 3 times. Added the 3,3-diaminobenzidine (DAB) chromogenic solution to develop color, put the slice into hematoxylin counterstain for 2 minutes, then dehydrated, transparentized, and sealed, and finally observed under the microscope.

\subsection{Statistical analysis}

In this study, Image-Pro Plus 6.0(IPP) software was used to analyze the integrated optical density (IOD) to quantify the expression of each indicator. SPSS 22.0 software was used for statistical analyses. All data were presented as means \pm standard deviation (SD). Differences between the two groups were analyzed using Student's t-test. Correlation between TRPC6 and NLRP3 expression levels was analyzed using the Pearson correlation test. To investigate whether NLRP3 inflammasome activation simultaneously mediates the HSPN development, a test of multiple mediations using the multiple-step mediator model was used ${ }^{28}$. Namely, the PROCESS plugin was installed into the IBM SPSS 22.0 software. The model number was 4, and bootstrap samples were 5000. Items "Bias Corrected" and "Compare indirect effects" were opted. $P$ values $<0.05$ were considered significant.

\section{Results}

\subsection{Comparison of TRPC6 expression and NLRP3 inflammasome related indicators}

In the normal control group, little NLRP3, ASC, Caspase-1, IL-1ß, IL-18 were observed. There was a little TRPC6 expression in mesangial cells and podocytes in the control group. The expression of NLRP3, ASC, 
Caspase-1, IL-1 $\beta$, IL-18, TRPC6 was significantly higher than that of the control group $(P<0.001)$. The positive products of NLRP3, ASC, Caspase-1, IL-1 $\beta$, IL-18, and TRPC6 were all brownish yellow fine particles observed under the microscope, and they were mainly located in the cytoplasm of glomerular cells, and the renal tubular cells were also expressed in large amounts (Table 1 and Figure 1).

Table 1. Comparison of TRPC6 expression and NLRP3 inflammasome related indicators for the two groups(IOD)

\begin{tabular}{|lllll|}
\hline Variables & Mean \pm SD & & \multirow{2}{*}{ tvalue } & $P$-value \\
\cline { 2 - 5 } & Control group & HSPN group & & \\
\hline NLRP3 & $17.92 \pm 5.64$ & $45.66 \pm 24.08$ & -5.798 & $<0.001$ \\
\hline ASC & $20.74 \pm 4.79$ & $66.43 \pm 29.95$ & -8.204 & $<0.001$ \\
\hline Caspase-1 & $14.11 \pm 5.56$ & $66.93 \pm 39.79$ & -7.245 & $<0.001$ \\
\hline IL-1 $\beta$ & $11.86 \pm 3.35$ & $66.81 \pm 37.12$ & -8.319 & $<0.001$ \\
\hline IL-18 & $17.70 \pm 1.31$ & $65.43 \pm 30.81$ & -8.885 & $<0.001$ \\
\hline TRPC6 & $12.29 \pm 4.20$ & $104.70 \pm 47.38$ & -10.969 & $<0.001$ \\
\hline
\end{tabular}

\subsection{Correlation between TRPC6 and NLRP3 inflammasome related indicators in the HSPN group}

In the HSPN group, the expression of TRPC6 was moderately related to the expression of NLRP3, ASC, and IL-18. Low to moderate correlation of NLRP3 with IL-1 $\beta$ and IL-18 was revealed. The expression level of ASC was moderately associated with the expression of IL-1 $\beta$ and IL-18 (Figure 2).

\subsection{Multiple mediation analysis results}

A separately run individual set of regressions indicated significant direct effects of TRPC6 on NLRP3 and ASC, effects of ASC on IL-1 $\beta$ and IL-18, effects of IL-10 on IL-18. However, only the mediation path from TRPC6 to ASC, and from ASC to HSPN remained significant. A 95\% confidence interval from 0.04 to 0.15 for the indirect effect (0.08) was revealed, explaining $58.25 \%$ of the total effect. All other mediation paths became insignificant(Figure 3).

\section{Discussion}

HSPN as the most serious complication of HSP, because the specific pathophysiology of its pathogenesis is not yet clear, its incidence has not decreased over time ${ }^{29}$. The results of this study showed that the expressions of TRPC6 and NLRP3 inflammasome in kidney tissues of children with HSPN were significantly higher than those in the normal control group $(P<0.05)$. The increased expression of TRPC6 in kidney tissues of HSPN children was correlated with the increased expression of NLRP3, ASC, and IL18. The expression of IL-1 $\beta$ and IL-18 was correlated with the expression of NLRP3, ASC. It indicated that 
the increased expression of TRPC6 and the activation of NLRP3 inflammasome do participate in the process of HSPN kidney injury.

TRPC6 is one of the newly discovered podocytes slit diaphragm proteins (SD). It interacts with a variety of SD molecules such as Podocin, Nephrin, and CD2AP to form a signaling complex to maintain the structural and functional integrity of glomerular podocytes ${ }^{30,31}$. TRPC6 expression is increased in a variety of acquired proteinuria glomerular diseases, which may be caused by regulating calcium influx ${ }^{32}$, 33. However, it is not very clear how TRPC6 is specifically involved in the pathogenesis of podocyte injury. Studies have shown that the upregulation of TRPC6 can be detected in various glomerular diseases such as minimal change nephropathy, membranous nephropathy, and FSGS ${ }^{34}$. Another study has found that TRPC6 is found in some secondary glomerular diseases such as diabetic nephropathy (DN) ${ }^{35}$. It also significantly increased the expression of glomerular podocyte TRPC6 in children with nephrotic syndrome ${ }^{36}$. Combined with the results of this study, it is not difficult to see that TRPC6 plays an important role in the progression of glomerular disease, and taking measures against it will greatly help the diagnosis and prevention of kidney disease. NLRP3 inflammasome is one of the members of the intracellular inflammatory protein complex family, which is composed of NLRP3 protein, adaptor ASC (an apoptosis-related speck-like protein containing CARD domain) and Caspase-1 precursor ${ }^{9}$. In normal cells, these protein complexes exist in an inactive form. After activation of the inflammatory body, the precursor protein of Caspase- 1 is cleaved into functional Caspase-1. Its main function is to convert the inactive and intracellular pro-inflammatory cytokines pro-IL-1 $\beta$ and pro-IL-18 It is converted into active IL-1 $\beta$ and IL-18 and released to the outside of the cell, causing local tissue inflammatory damage or systemic inflammatory response ${ }^{37,38}$. Zhang used confocal microscopy to observe the expression of NLRP3 and ASC in glomerular podocytes in vitro ${ }^{39}$. At the same time, studies have found that the amount of NLRP3 protein and its mRNA will be significantly increased in kidney diseases, such as IgA nephropathy ${ }^{40}$, hypertensive nephropathy ${ }^{41}$, crescentic nephritis ${ }^{42}$, and acute tubular necrosis ${ }^{43}$. Although few studies have reported on the role of NLRP3 inflammasome in the occurrence and development of HSPN. Combining the above research results with this research results, we can see that the NLRP3 inflammasome is a key protein complex of kidney inflammation and is inextricably linked to kidney disease.

Studies have shown that Ang $₫$ can quickly activate the TRPC6 channel of podocytes and mediate calcium influx, and at the same time cause a significant upregulation of TRPC6 activity and expression 22 , 23. Ang $₫$ can increase the level of TRPC6 channel protein expression, increase podocyte calcium influx, activate calcineurin and stimulate NFAT dephosphorylation, at the same time upregulate the expression of cytokines, stimulate extracellular matrix (ECM) production, activate multiple cells internal signal pathway leads to cell damage ${ }^{35,44}$. The NLRP3 inflammasome is one of the intracellular inflammasomes. When the body senses an external stimulus, the stimulus signal enters the cell, activates NLRP3, recruits ASC, and Pro-Caspase1 to form the NLRP3 inflammasome, generates activated Caspase1 , cleaves the inflammatory factors precursor and activated inflammatory factors IL-1 $\beta$ and IL18 are 
produced, and cell efflux leads to inflammation. It is currently believed that various NLRP3 inflammasome stimulants can cause mitochondrial dysfunction, leading to the release of reactive oxygen species ROS and $\mathrm{mtDNA}$, thereby activating the NLRP3 inflammasome. $\mathrm{Ca}^{2+}$ released into the cytoplasm under endoplasmic reticulum stress can be taken up by the mitochondrial unidirectional transporter, which in turn reduces the normal negative mitochondrial transmembrane potential, activates the mitochondrial outer membrane voltage-gated channel, promotes related metabolites and ion transport and produce ROS, activates the NLRP3 inflammasome. When the voltage on the outer membrane of the mitochondria is knocked down, the generation of ROS is reduced, and the activation of the NLRP3 inflammasome is impaired $^{45}$. A report ${ }^{46}$ believed that oxidized mtDNA released by dysfunctional mitochondria is also associated with the activation of NLRP3 inflammasome. They found that the a7-nicotinic acetylcholine receptor signaling pathway can inhibit the activation of the NLRP3 inflammasome by blocking the release of mtDNA. Recently, more and more researchers have begun to pay attention to the role of $\mathrm{Ca}^{2+}$ in the activation of NLRP3 inflammasome. Extracellular $\mathrm{Ca}^{2+}$ influx or $\mathrm{Ca}^{2+}$ release from the intracellular calcium pool to the cytoplasm and cause mitochondrial dysfunction is a common pathway for multiple activators to activate the NLRP3 inflammasome ${ }^{26}$. Based on the results of this study, it is reasonable to infer that: TRPC6 channel-mediated calcium influx may release ROS and mtDNA through mitochondrial damage and participate in the activation of NLRP3 inflammatory bodies, producing free IL-1 $\beta$ and IL-18, and participating in HSPN kidney damage process (Figure 4).

To our knowledge, this is the first time to investigate the relationship between expression levels of TRPC6 and NLRP3 inflammasome in kidney tissue of children with HSPN. Some limitations should be considered. First, the sample size of this study recruiting 33 cases and 6 controls was small, with the statistical power is limited. Second, demographic characteristics as well as other covariates were not abstracted. Other indicators including Ang $\otimes$ and ROS expression levels were not detected because of limited samples and funding. Finally, only the statistical association between TRPC6 and NLRP3 was observed. The specific mechanism pathway should be further explored by animal experiments and in vitro cell experiments. This study also did not delve into the effects of changes in the molecular structure of TRPC6 and NLRP3 inflammasome on the disease. The main objective was to propose a hypothesis based on findings of this study, to provide the guidelines for our subsequent cell experimental research.

\section{Conclusion}

In summary, TRPC6 and NLRP3 inflammasome play an important role in the pathogenesis of kidney disease in children with HSPN. TRPC 6 may cause the release of IL-1 $\beta$ and IL-18 by activating the NLRP3 inflammasome, especially the expression of ASC, thereby damaging the kidneys of children with HSP. These new findings were conducive to further study the mechanism of kidney injury in children with HSPN, the specific interaction mechanism between TRPC6 and NLRP3 inflammasome in kidney tissues, and then as a molecular target for the targeted treatment of HSPN.

\section{Abbreviations}




\begin{tabular}{|ll|}
\hline Abbreviation & Full name \\
\hline HSPN & Henoch-Schönlein purpura nephritis \\
\hline TRPC6 & transient receptor potential cation channel protein 6 \\
\hline NLRP3 & Nod-like receptor protein 3 \\
\hline ASC & apoptosis-related speck-like protein containing CARD domain \\
\hline IL-1 $\beta$ & Interleukin-1 $\beta$ \\
\hline IL-18 & Interleukin-18 \\
\hline HSP & Henoch-Schönlein purpura \\
\hline ROS & reactive oxygen species \\
\hline mtDNA & mitochondrial DNA \\
\hline AKI & Acute kidney injury \\
\hline TRP & Transient receptor potential \\
\hline FSGS & familial forms of focal segmental glomerulosclerosis \\
\hline Ang $\bigotimes$ & angiotensin $\bigotimes$ \\
\hline IHC & Immunohistochemistry \\
\hline DAB & 3,3-diaminobenzidine \\
\hline PBS & phosphate buffered saline \\
\hline IOD & integrated optical density \\
\hline SD & slit diaphragm proteins \\
\hline DN & diabetic nephropathy \\
\hline ECM & \\
\hline
\end{tabular}

\section{Declarations}

\section{Ethics approval and consent to participate}

The study was approved by the Medical Research Ethics Committee of the First Affiliated Hospital of Anhui Medical University, informed consent was obtained from all guardians of individual participants included in the study.

\section{Consent for publication}


Our data have consent for publication from that person, or in the case of children, their parent or legal guardian.

\section{Availability of data and materials}

The datasets used and/or analysed during the current study are available from the corresponding author on reasonable request.

\section{Competing interests}

The authors declare that they have no competing interests.

\section{Funding}

The study was funded by the 2019 Doctoral Research Fund from the first affiliated Hospital of Anhui Medical University (BSKY2019027); Anhui Province 2017 Public Welfare Technology Application Research Linkage Plan Project (1704f0804027); Anhui Provincial Department of Education 2017 College Outstanding Top Talent Training Project (gxbjZD07).

\section{Authors' contributions}

$\mathrm{XH}$ W performed the histological examination of the kidney, and was a major contributor in writing the manuscript. M W collected statistics about children. Y L collected statistical information on immunohistochemistry results and reviewed the manuscript. $\mathrm{H} \mathrm{G}$ analyzed the patient data and reviewed and edited the manuscript. F D provided experimental design ideas and fund support, and supervised the publication of this article as the corresponding author. All authors read and approved the final manuscript.

\section{Acknowledgements}

This study was supported by the First Affiliated Hospital of Anhui Medical University and the Key Laboratory Zoonses of Anhui Province.

\section{References}

1. Zhang Q, Yan L, Chen M, Gui M, Lu L, Deng F, et al. IgA1 isolated from Henoch-Schonlein purpura children promotes proliferation of human mesangial cells in vitro. Cell Biol Int. 2019;43(7):760-769.

2. Gardner-Medwin JM, Dolezalova P, Cummins C, Southwood TR. Incidence of Henoch-Schonlein purpura, Kawasaki disease, and rare vasculitides in children of different ethnic origins. Lancet. 2002;360(9341):1197-1202.

3. Chen JY, Mao JH. Henoch-Schonlein purpura nephritis in children: incidence, pathogenesis and management. World J Pediatr. 2015;11(1):29-34. 
4. Narchi H. Risk of long term renal impairment and duration of follow up recommended for HenochSchonlein purpura with normal or minimal urinary findings: a systematic review. Arch Dis Child. 2005;90(9):916-920.

5. Hou JH, Zhu HX, Zhou ML, Le WB, Zeng CH, Liang SS, et al. Changes in the Spectrum of Kidney Diseases: An Analysis of 40,759 Biopsy-Proven Cases from 2003 to 2014 in China. Kidney Dis (Basel). 2018;4(1):10-19.

6. He Y, Hara H, Nunez G. Mechanism and Regulation of NLRP3 Inflammasome Activation. Trends Biochem Sci. 2016;41(12):1012-1021.

7. Liu Y, Xu Z, Ma F, Jia Y, Wang G. Knockdown of TLR4 attenuates high glucose-induced podocyte injury via the NALP3/ASC/Caspase-1 signaling pathway. Biomed Pharmacother. 2018;107:13931401.

8. Wang C, Hou XX, Rui HL, Li LJ, Zhao J, Yang M, et al. Artificially Cultivated Ophiocordyceps sinensis Alleviates Diabetic Nephropathy and Its Podocyte Injury via Inhibiting P2X7R Expression and NLRP3 Inflammasome Activation. J Diabetes Res. 2018;2018:1390418.

9. Pilot T, Fratti A, Thinselin C, Perrichet A, Demontoux L, Limagne E, et al. Heat shock and HSP70 regulate 5-FU-mediated caspase-1 activation in myeloid-derived suppressor cells and tumor growth in mice. J Immunother Cancer. 2020;8(1).

10. Zhou R, Tardivel A, Thorens B, Choi I, Tschopp J. Thioredoxin-interacting protein links oxidative stress to inflammasome activation. Nat Immunol. 2010;11(2):136-140.

11. Munoz-Planillo R, Kuffa P, Martinez-Colon G, Smith BL, Rajendiran TM, Nunez G. K(+) efflux is the common trigger of NLRP3 inflammasome activation by bacterial toxins and particulate matter. Immunity. 2013;38(6):1142-1153.

12. Komada T, Muruve DA. The role of inflammasomes in kidney disease. Nat Rev Nephrol. 2019;15(8):501-520.

13. Wen Y, Liu YR, Tang TT, Pan MM, Xu SC, Ma KL, et al. mROS-TXNIP axis activates NLRP3 inflammasome to mediate renal injury during ischemic AKI. Int J Biochem Cell Biol. 2018;98:43-53.

14. Subramanian N, Natarajan K, Clatworthy MR, Wang Z, Germain RN. The adaptor MAVS promotes NLRP3 mitochondrial localization and inflammasome activation. Cell. 2013;153(2):348-361.

15. Komada T, Usui F, Kawashima A, Kimura H, Karasawa T, Inoue Y, et al. Role of NLRP3 Inflammasomes for Rhabdomyolysis-induced Acute Kidney Injury. Sci Rep. 2015;5:10901.

16. Lau A, Chung H, Komada T, Platnich JM, Sandall CF, Choudhury SR, et al. Renal immune surveillance and dipeptidase-1 contribute to contrast-induced acute kidney injury. J Clin Invest. 2018;128(7):28942913.

17. Reiser J, Polu KR, Moller CC, Kenlan P, Altintas MM, Wei C, et al. TRPC6 is a glomerular slit diaphragm-associated channel required for normal renal function. Nat Genet. 2005;37(7):739-744.

18. Sours S, Du J, Chu S, Ding M, Zhou XJ, Ma R. Expression of canonical transient receptor potential (TRPC) proteins in human glomerular mesangial cells. Am J Physiol Renal Physiol. 2006;290(6):F1507-1515. 
19. Dryer SE, Roshanravan H, Kim EY. TRPC channels: Regulation, dysregulation and contributions to chronic kidney disease. Biochim Biophys Acta Mol Basis Dis. 2019;1865(6):1041-1066.

20. Li T, Deng F, Zhang X-c, Zhao Q-x, Li X, Zhang H-I (2017) Role of angiotensinogen in progression of Henoch-Schönlein purpura nephritis in children. China Journal of Modern Medicine 27:53-57. (Chinese)

21. Jang HR, Kim SM, Lee YJ, Lee JE, Huh W, Kim DJ, et al. The origin and the clinical significance of urinary angiotensinogen in proteinuric IgA nephropathy patients. Ann Med. 2012;44(5):448-457.

22. Huang H, You Y, Lin X, Tang C, Gu X, Huang M, et al. Inhibition of TRPC6 Signal Pathway Alleviates Podocyte Injury Induced by TGF-beta1. Cell Physiol Biochem. 2017;41(1):163-172.

23. Ilatovskaya DV, Palygin O, Chubinskiy-Nadezhdin V, Negulyaev YA, Ma R, Birnbaumer L, et al. Angiotensin II has acute effects on TRPC6 channels in podocytes of freshly isolated glomeruli. Kidney Int. 2014;86(3):506-514.

24. An X, Sun X, Yang X, Liu D, Hou Y, Chen H, et al. Oxidative stress promotes ventilator-induced lung injury through activating NLRP3 inflammasome and TRPM2 channel. Artif Cells Nanomed Biotechnol. 2019;47(1):3448-3455.

25. Zheng Q, Tan Q, Ren Y, Reinach PS, Li L, Ge C, et al. Hyperosmotic Stress-Induced TRPM2 Channel Activation Stimulates NLRP3 Inflammasome Activity in Primary Human Corneal Epithelial Cells. Invest Ophthalmol Vis Sci. 2018;59(8):3259-3268.

26. Horng T. Calcium signaling and mitochondrial destabilization in the triggering of the NLRP3 inflammasome. Trends Immunol. 2014;35(6):253-261.

27. Zhu C, Huang S (2017) Evidence-Based Guidelines for the Diagnosis and Treatment of HenochSchönlein purpura Nephritis (2016). Chin J Pediatr 55:647-651.(Chinese)

28. Preacher KJ, Hayes AF. Asymptotic and resampling strategies for assessing and comparing indirect effects in multiple mediator models. Behav Res Methods. 2008;40(3):879-891.

29. Kawasaki Y, Suyama K, Yugeta E, Katayose M, Suzuki S, Sakuma H, et al. The incidence and severity of Henoch-Schonlein purpura nephritis over a 22-year period in Fukushima Prefecture, Japan. Int Urol Nephrol. 2010;42(4):1023-1029.

30. Hsu YJ, Hoenderop JG, Bindels RJ. TRP channels in kidney disease. Biochim Biophys Acta. 2007;1772(8):928-936.

31. Wieder N, Greka A. Calcium, TRPC channels, and regulation of the actin cytoskeleton in podocytes: towards a future of targeted therapies. Pediatr Nephrol. 2016;31(7):1047-1054.

32. Ilatovskaya DV, Staruschenko A. TRPC6 channel as an emerging determinant of the podocyte injury susceptibility in kidney diseases. Am J Physiol Renal Physiol. 2015;309(5):F393-397.

33. Szabo T, Ambrus L, Zakany N, Balla G, Biro T. Regulation of TRPC6 ion channels in podocytes Implications for focal segmental glomerulosclerosis and acquired forms of proteinuric diseases. Acta Physiol Hung. 2015;102(3):241-251. 
34. Riehle M, Buscher AK, Gohlke BO, Kassmann M, Kolatsi-Joannou M, Brasen JH, et al. TRPC6 G757D Loss-of-Function Mutation Associates with FSGS. J Am Soc Nephrol. 2016;27(9):2771-2783.

35. Sonneveld R, van der Vlag J, Baltissen MP, Verkaart SA, Wetzels JF, Berden JH, et al. Glucose specifically regulates TRPC6 expression in the podocyte in an Angll-dependent manner. Am J Pathol. 2014;184(6):1715-1726.

36. Sheng H, WU Ying, Weixun H, Yulin K, Wenyan H, Guanghua Z (2017) Expression of TRPC 6 in renal tissue and its significance in children with primary nephrotic syndrome. J Clin Pediatr 35:498-502. (Chinese)

37. Martine P, Chevriaux A, Derangere V, Apetoh L, Garrido C, Ghiringhelli F, et al. HSP70 is a negative regulator of NLRP3 inflammasome activation. Cell Death Dis. 2019;10(4):256.

38. Martine P, Rebe C. Heat Shock Proteins and Inflammasomes. Int J Mol Sci. 2019;20(18).

39. Zhang C, Boini KM, Xia M, Abais JM, Li X, Liu Q, et al. Activation of Nod-like receptor protein 3 inflammasomes turns on podocyte injury and glomerular sclerosis in hyperhomocysteinemia. Hypertension. 2012;60(1):154-162.

40. Tsai YL, Hua KF, Chen A, Wei CW, Chen WS, Wu CY, et al. NLRP3 inflammasome: Pathogenic role and potential therapeutic target for IgA nephropathy. Sci Rep. 2017;7:41123.

41. Wen Y, Liu Y, Tang T, Lv L, Liu H, Ma K, et al. NLRP3 inflammasome activation is involved in Ang IIinduced kidney damage via mitochondrial dysfunction. Oncotarget. 2016;7(34):54290-54302.

42. Lichtnekert J, Kulkarni OP, Mulay SR, Rupanagudi KV, Ryu M, Allam R, et al. Anti-GBM glomerulonephritis involves IL-1 but is independent of NLRP3/ASC inflammasome-mediated activation of caspase-1. PLoS One. 2011;6(10):e26778.

43. Xiao YD, Huang YY, Wang HX, Wu Y, Leng Y, Liu M, et al. Thioredoxin-Interacting Protein Mediates NLRP3 Inflammasome Activation Involved in the Susceptibility to Ischemic Acute Kidney Injury in Diabetes. Oxid Med Cell Longev. 2016;2016:2386068.

44. Wennmann DO, Hsu HH, Pavenstadt H. The renin-angiotensin-aldosterone system in podocytes. Semin Nephrol. 2012;32(4):377-384.

45. Shenker BJ, Ojcius DM, Walker LP, Zekavat A, Scuron MD, Boesze-Battaglia K. Aggregatibacter actinomycetemcomitans cytolethal distending toxin activates the NLRP3 inflammasome in human macrophages, leading to the release of proinflammatory cytokines. Infect Immun. 2015;83(4):14871496.

46. Lu B, Kwan K, Levine YA, Olofsson PS, Yang H, Li J, et al. alpha7 nicotinic acetylcholine receptor signaling inhibits inflammasome activation by preventing mitochondrial DNA release. $\mathrm{Mol}$ Med. 2014;20:350-358.

\section{Figures}



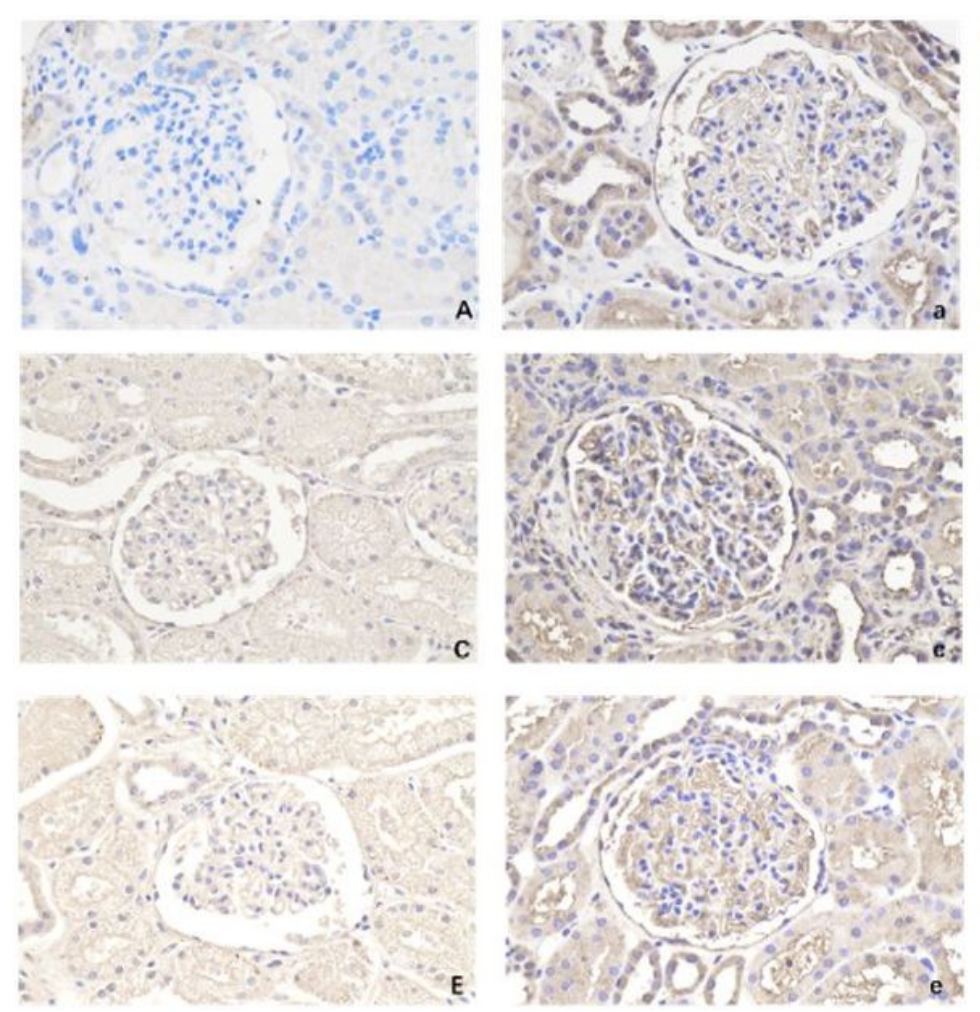
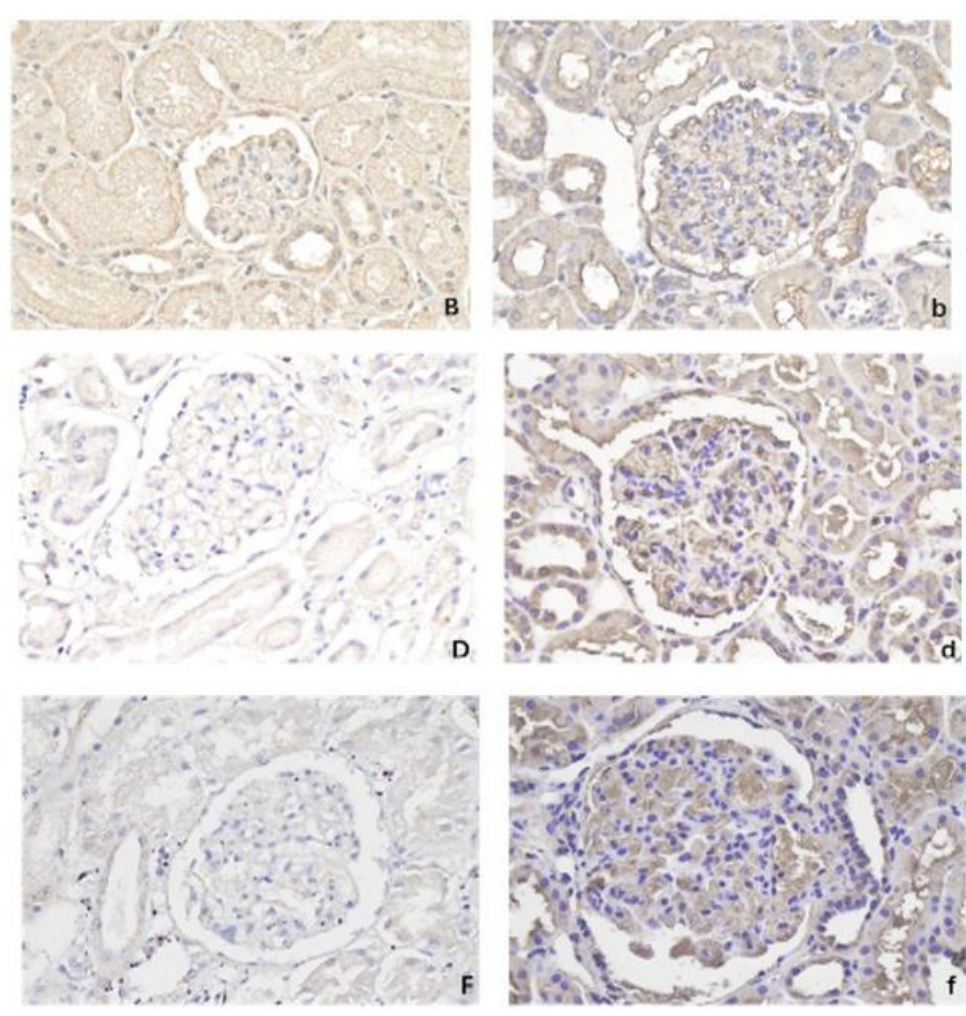

\section{Figure 1}

Expression of TRPC6 and NLRP3 inflammasome related indicators in kidney tissues of two groups A: Control group NLRP3; a: HSPN group NLRP3; B: control group ASC; b: HSPN group ASC; C: control group Caspase-1; c: HSPN group Caspase-1; D: Control group IL-1 $\beta$; d: HSPN group IL-1 $\beta$; E: control group IL-18; e: HSPN group IL-18; F: control group TRPC6; f: HSPN group TRPC6.
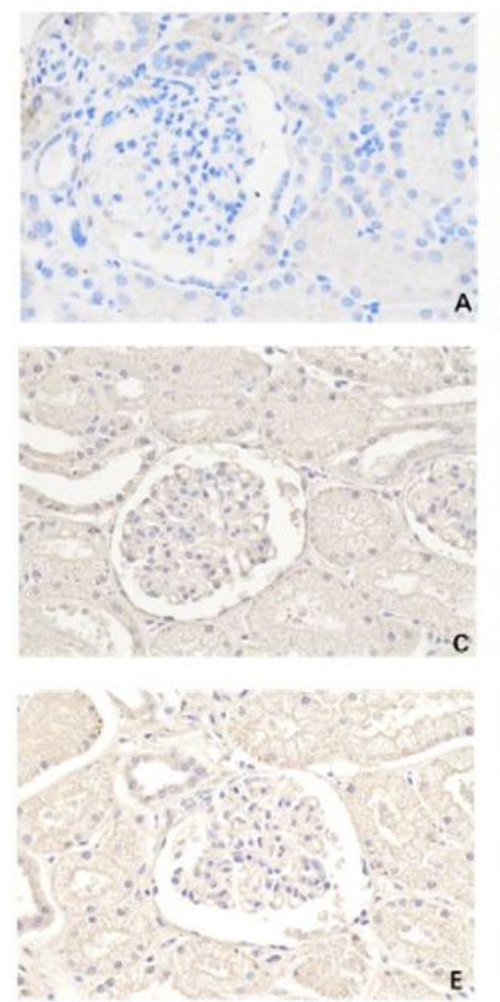
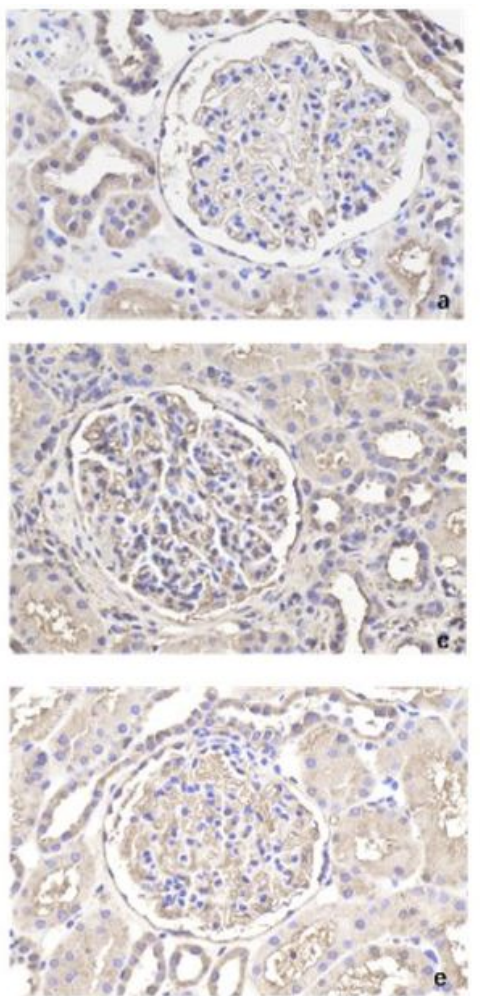
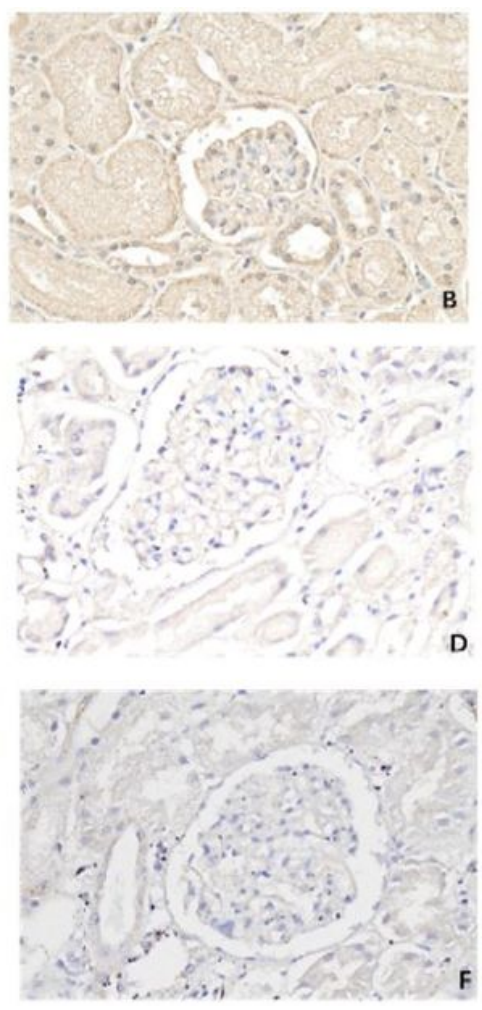
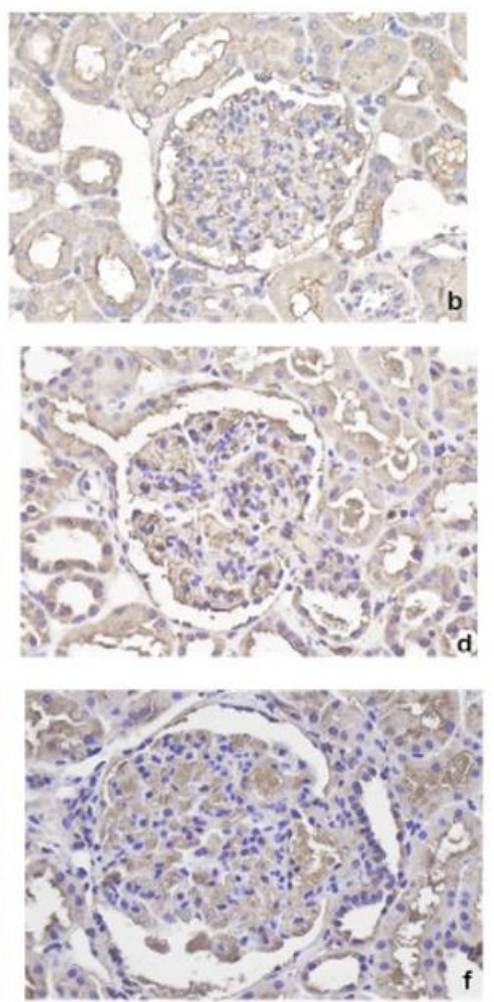


\section{Figure 1}

Expression of TRPC6 and NLRP3 inflammasome related indicators in kidney tissues of two groups A: Control group NLRP3; a: HSPN group NLRP3; B: control group ASC; b: HSPN group ASC; C: control group Caspase-1; c: HSPN group Caspase-1; D: Control group IL-1 $\beta$; d: HSPN group IL-1 $\beta$; E: control group IL-18; e: HSPN group IL-18; F: control group TRPC6; f: HSPN group TRPC6.

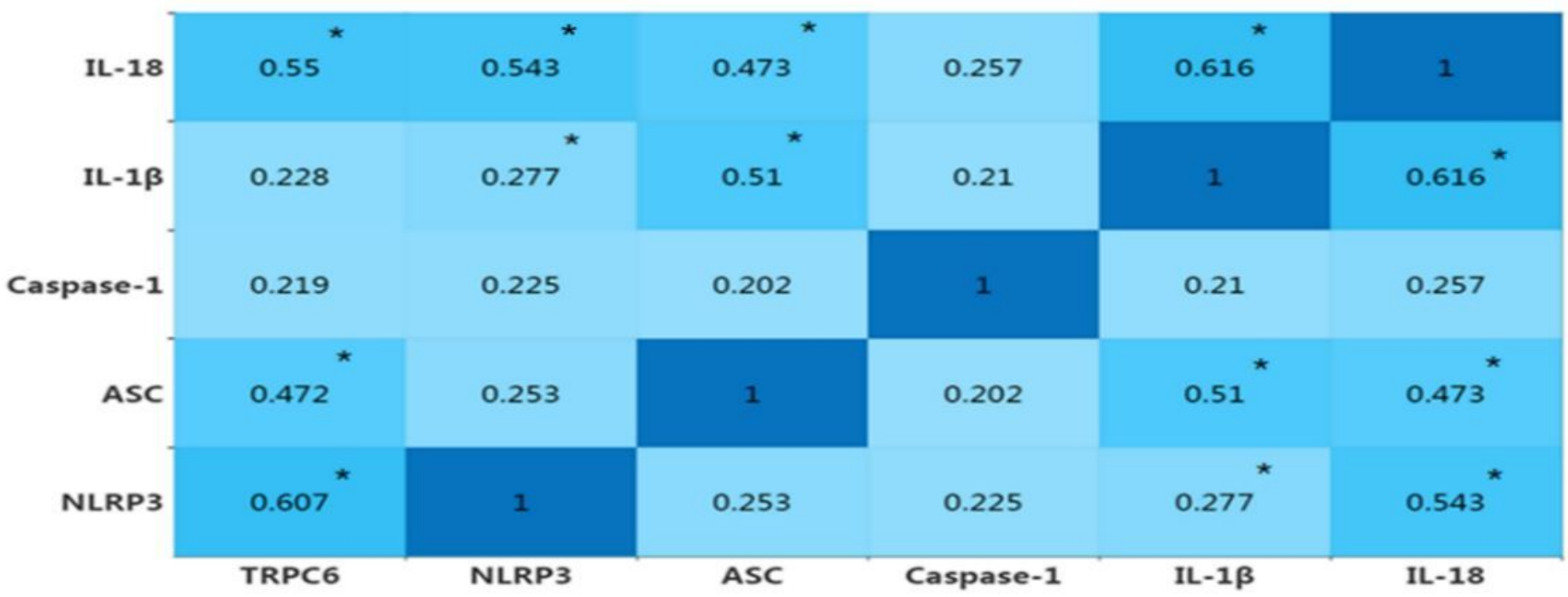

\section{Figure 2}

Correlation between TRPC6 and NLRP3 inflammasome related indicators among the HSPN group ( ${ }^{*}$ $<0.05)$

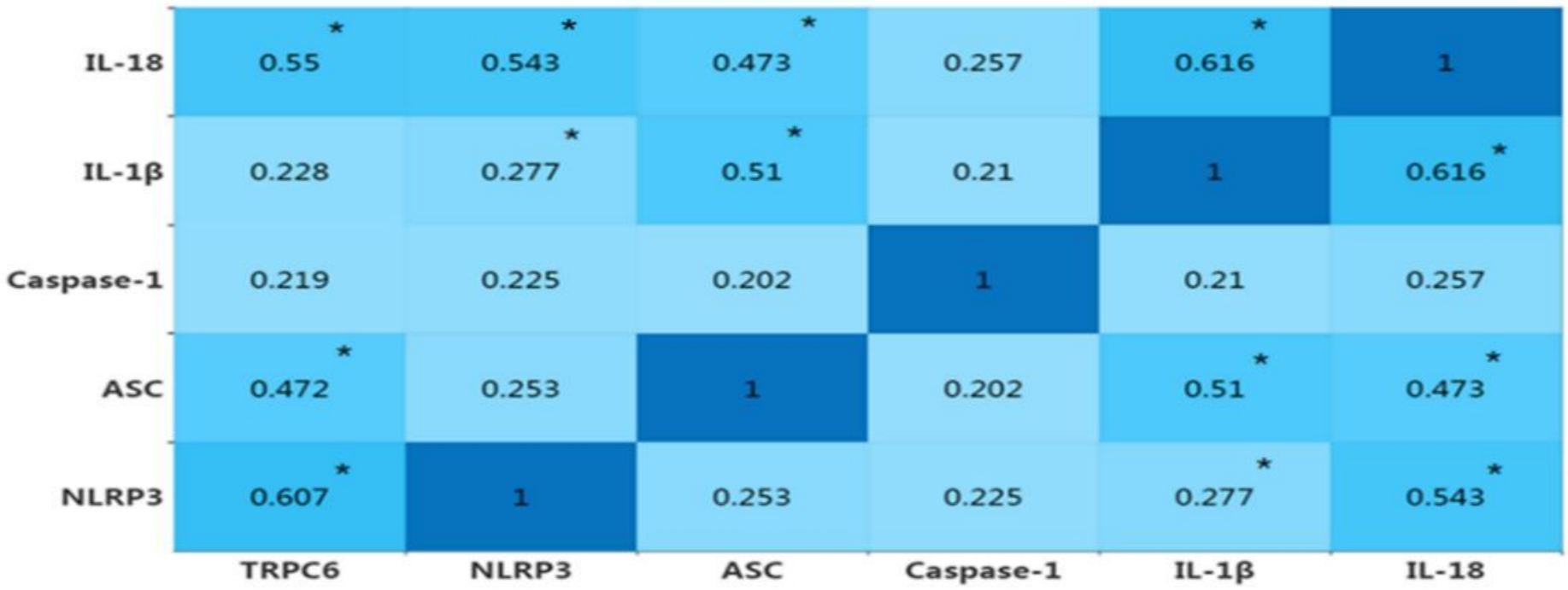

Figure 2

Correlation between TRPC6 and NLRP3 inflammasome related indicators among the HSPN group ( ${ }^{*}$ $<0.05$ ) 


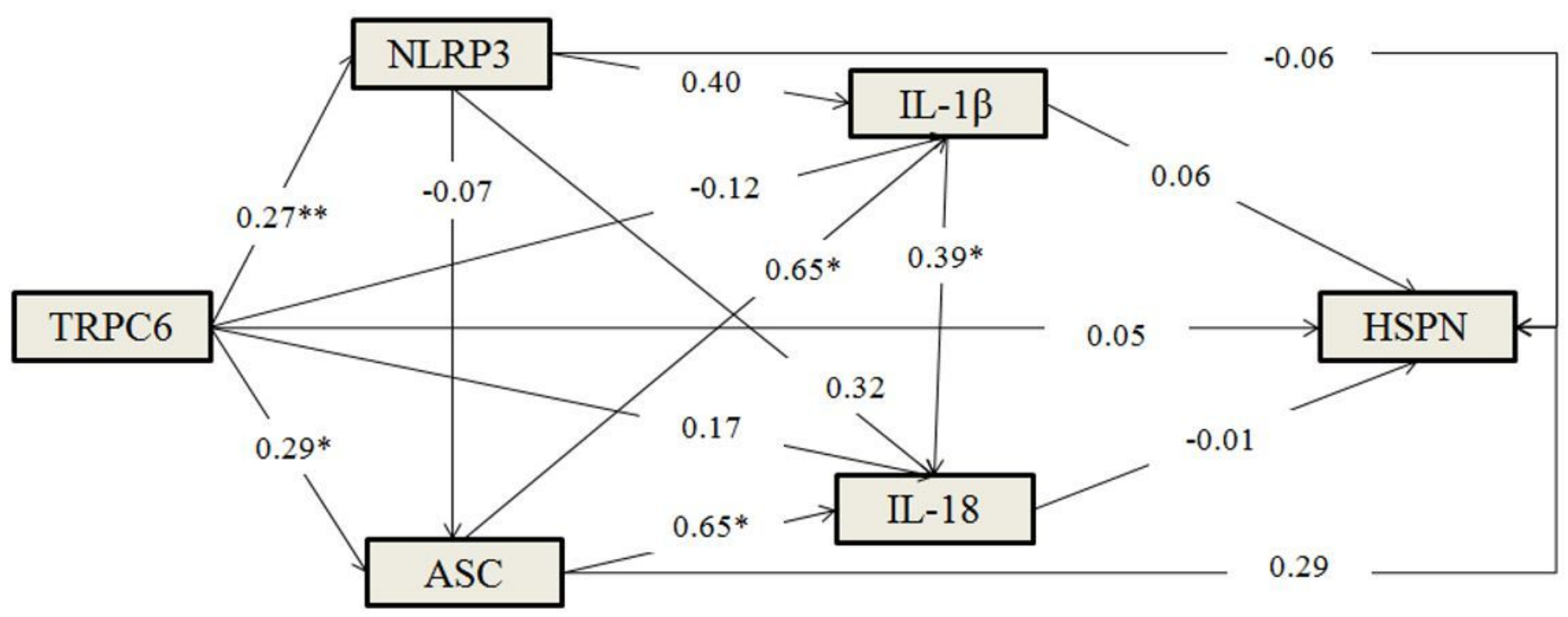

Figure 3

Multistep multiple-mediation model of the effect of TRPC6 and NLRP3 inflammasome on HSPN development (Coefficients are unstandardized. ${ }^{*} \mathrm{P}<0.05$, ${ }^{*} \mathrm{P}<0.01$ )

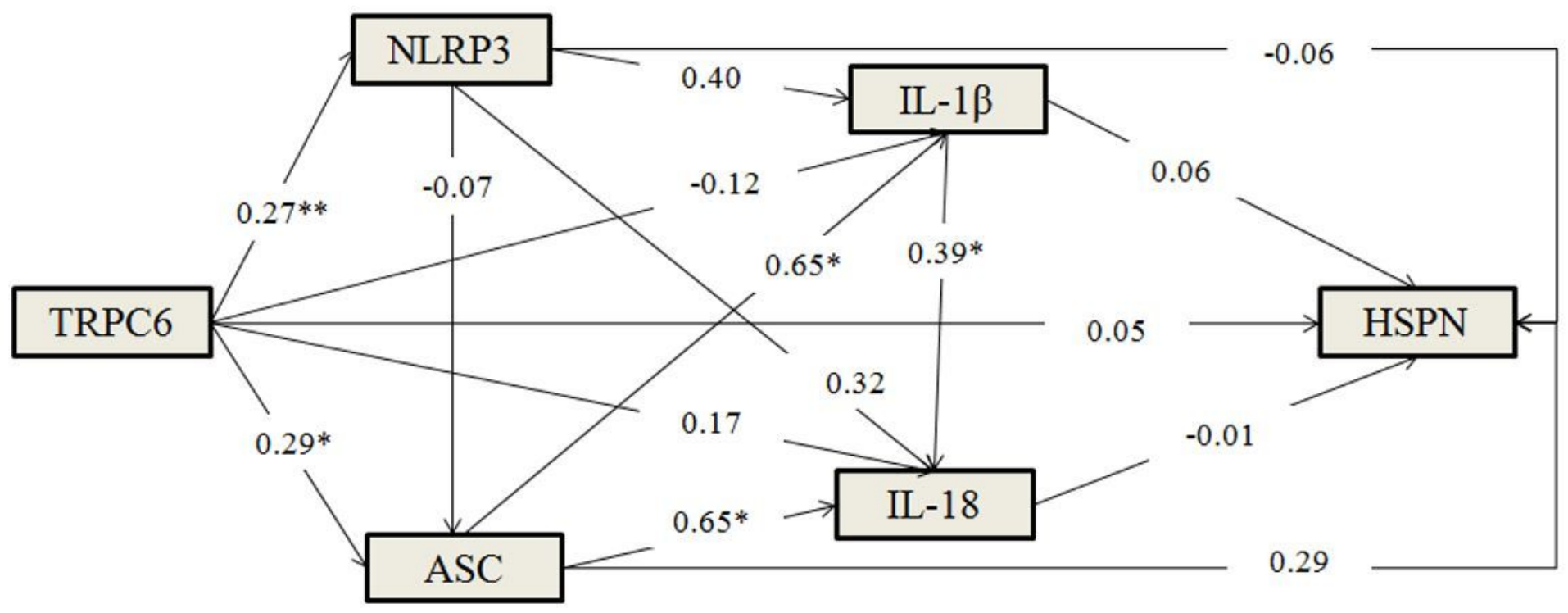

\section{Figure 3}

Multistep multiple-mediation model of the effect of TRPC6 and NLRP3 inflammasome on HSPN development (Coefficients are unstandardized. ${ }^{*} \mathrm{P}<0.05,{ }^{*} \mathrm{P}<0.01$ ) 


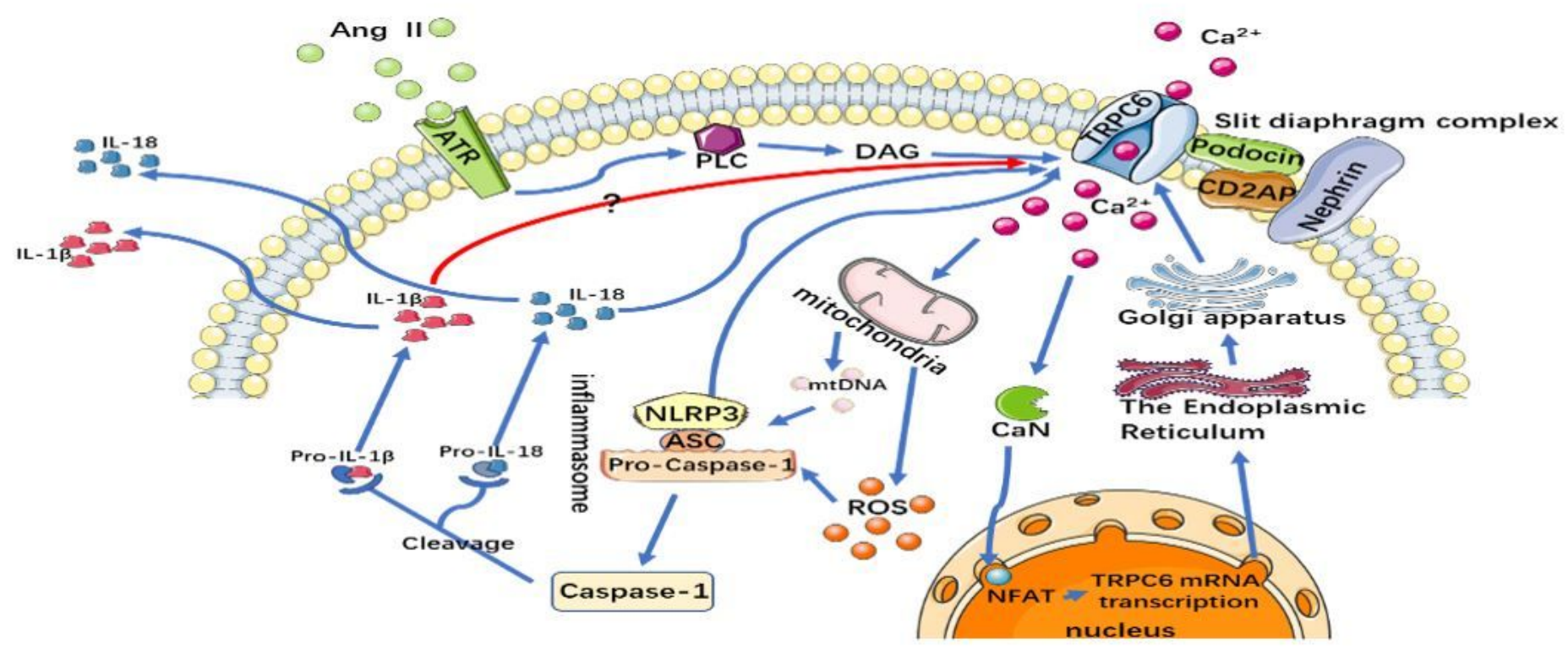

Figure 4

Hypothesis diagram of TRPC6 and NLRP3 inflammasome activation pathway

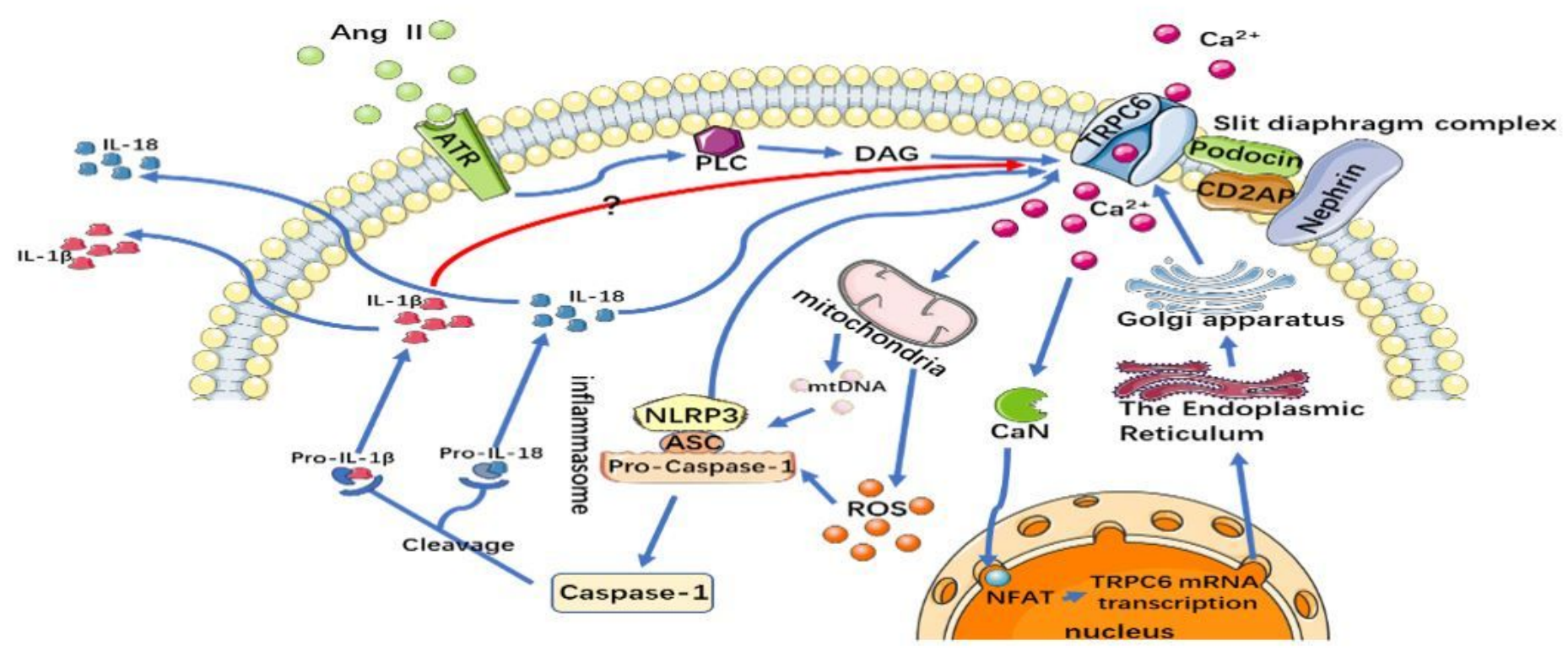

Figure 4

Hypothesis diagram of TRPC6 and NLRP3 inflammasome activation pathway 